Review Article

\title{
The Dual Role of Nrf2 in Nonalcoholic Fatty Liver Disease: Regulation of Antioxidant Defenses and Hepatic Lipid Metabolism
}

\author{
Sílvia S. Chambel, ${ }^{1,2}$ Andreia Santos-Gonçalves, ${ }^{1,2}$ and Tiago L. Duarte ${ }^{1,2}$ \\ ${ }^{1}$ Basic and Clinical Research on Iron Biology Group, Instituto de Biologia Molecular e Celular (IBMC), Universidade do Porto, \\ Rua do Campo Alegre 823, 4150180 Porto, Portugal \\ ${ }^{2}$ Instituto de Investigação e Inovação em Saúde, Universidade do Porto, Portugal
}

Correspondence should be addressed to Tiago L. Duarte; tduarte@ibmc.up.pt

Received 10 October 2014; Revised 16 January 2015; Accepted 19 January 2015

Academic Editor: Maria Carmen Collado

Copyright @ 2015 Sílvia S. Chambel et al. This is an open access article distributed under the Creative Commons Attribution License, which permits unrestricted use, distribution, and reproduction in any medium, provided the original work is properly cited.

\begin{abstract}
Nonalcoholic fatty liver disease (NAFLD) is a progressive liver disease with ever-growing incidence in the industrialized world. It starts with the simple accumulation of lipids in the hepatocyte and can progress to the more severe nonalcoholic steatohepatitis (NASH), which is associated with inflammation, fibrosis, and cirrhosis. There is increasing awareness that reactive oxygen species and electrophiles are implicated in the pathogenesis of NASH. Transcription factor nuclear factor erythroid 2-related factor 2 (Nrf2) is a positive regulator of the expression of a battery of genes involved in the protection against oxidative/electrophilic stress. In rodents, Nrf2 is also known to participate in hepatic fatty acid metabolism, as a negative regulator of genes that promote hepatosteatosis. We review relevant evidence in the literature that these two mechanisms may contribute to the protective role of Nrf2 in the development of hepatic steatosis and in the progression to steatohepatitis, particularly in young animals. We propose that age may be a key to explain contradictory findings in the literature. In summary, Nrf2 mediates the crosstalk between lipid metabolism and antioxidant defense mechanisms in experimental models of NAFLD, and the nutritional or pharmacological induction of Nrf2 represents a promising potential new strategy for its prevention and treatment.
\end{abstract}

\section{Hepatic Lipid Metabolism}

The liver plays a key role in the processing of lipids, including the synthesis and degradation/oxidation of fatty acids (FA), and the metabolism of cholesterol and phospholipids. Hepatocytes convert the excess dietary glucose into FAs (lipogenesis), which can be stored as triglycerides (TGs) in lipid droplets or used in the generation of phospholipids $[1,2]$. Lipogenesis involves acetyl-CoA precursors and is an insulindependent process. Under normal conditions, TGs along with cholesterol and phospholipids are assembled into very low density lipoprotein (VLDL) particles that can be secreted into the bloodstream for storage in other tissues in the form of lipid droplets, thus preventing TG accumulation in the cytoplasm of hepatocytes $[1,3]$. On the other hand, when the available glucose cannot meet the energy demands, hepatocytes break down TGs and cholesterol stored in lipid droplets through a lysosomal degradative pathway of macroautophagy designated lipophagy. The breakdown of TGs by lipophagy supplies free fatty acids (FFAs) required to sustain rates of mitochondrial $\beta$-oxidation for the generation of ATP [4]. FA degradation occurs also in peroxisomes ( $\beta$-oxidation) and in the endoplasmatic reticulum (ER) ( $\omega$-oxidation).

When fatty acid input exceeds the capacity of $\beta$-oxidation, accumulating acyl-CoA is drained by triglyceride synthesis, leading to the supraphysiological accumulation of fat in hepatocytes (hepatosteatosis), which is a hallmark of both alcoholic and nonalcoholic fatty liver disease (NAFLD) [5]. Hepatic steatosis increases the oxidation of FFAs and the rate of the tricarboxylic acid cycle. Increased FFA $\beta$-oxidation results in increased rates of electron leakage from the mitochondrial respiratory chain [6], resulting in higher free radical formation, and increases hydrogen peroxide production in the peroxisomes. A decrease in the mitochondrial quinone 
pool and the associated reduction of mitochondrial oxidation metabolism were also proposed to account for the increased mitochondrial reactive oxygen species (ROS) production under high-fat diet (HFD) [7]. Microsomal oxidation also contributes to oxidative stress via the activity of cytochromes P450 2E1 and P450 4A. The incorporation of free saturated fatty acids, the first products of de novo lipogenesis, into membrane phospholipids is detrimental to the ER and leads to $\mathrm{Ca}^{2+}$ release, which has the potential to injure adjacent mitochondria and promote apoptotic cell death (lipoapoptosis) $[2,8]$.

\section{NAFLD: Prevalence and Etiology}

The increase in obesity has become an alarming public health trend in the industrialized world, with a growing burden on health care costs $[9,10]$. Obesity leads to an increased propensity for the development of a complex array of factors that increase the risk to develop cardiovascular disease or type 2 diabetes, which have been termed the metabolic syndrome (abdominal obesity, atherogenic dyslipidemia, hypertension, and insulin resistance) [11]. NAFLD is the hepatic manifestation of the metabolic syndrome. In western countries, NAFLD is estimated to affect $20-30 \%$ of the general population, although the incidence may be even higher in obese individuals. The disease is highly correlated to obesity. It starts with the relatively benign accumulation of lipids in the hepatocyte. In up to one-third of patients, steatosis can progress to the more severe nonalcoholic steatohepatitis (NASH), which is associated with inflammation, fibrosis, and impaired liver function (cirrhosis) [12].

The detailed pathological mechanisms leading to the transition from lipid deposition to necroinflammation and cytotoxicity remain unclear, but a "two-hit" theory is widely advocated in the literature [14]. The first hit corresponds to "simple" hepatocellular lipid accumulation (steatosis) resulting from increased inflow of FFAs derived from insulin resistant adipose tissue, increased hepatic de novo lipogenesis, or impaired lipid export from hepatocytes. The second hit may be a consequence of increased ROS production in the liver resulting from the increased metabolism of FAs, resulting in mitochondrial dysfunction, upregulation of proinflammatory cytokines, and activation of hepatic stellate cells, which begin to proliferate and increase production of collagen, causing fibrosis. There is nevertheless growing evidence that the development of NAFLD and the progression to NASH are more complex processes than initially predicted by the two-hit theory. For instance, while hepatic TG formation is an early indicator of liver metabolic stress and disease, it is unlikely to be the initiating step in NASH. Instead, TGs may represent harmless storage compartments that divert FFAs away from potentially toxic pathways (e.g., microsomal oxidation), thus protecting hepatocytes from lipoapoptosis $[5,8,15]$. Moreover, multiple mechanisms/factors may contribute to the progression of steatosis to NASH (second hit), including increased ROS production in the liver (via activation of inflammatory cells, mitochondrial dysfunction, or the uncoupling of cytochrome P450 2E1 and 4A isoenzymes), the failure of hepatocytes to synthesize sufficient endogenous antioxidants, ER stress, liver and adipose tissue inflammation, decreased autophagic function, and/or the mild hepatic iron overload that is frequently seen in NAFLD patients $[8,16,17]$.

\section{Nrf2 Activation by Hepatic Oxidants/Electrophiles}

The liver has relatively high metabolic activity and is the main organ responsible for the biotransformation and subsequent detoxification of xenobiotics. These properties place the organ at an increased risk for exposure to ROS [2] and electrophiles [30] which, in turn, are increasingly implicated in the pathogenesis of NAFLD and other chronic liver diseases. In the hepatocyte, the major sites of ROS production are the mitochondria and the cytochrome P450 system. Electrophiles are produced by oxidation and nitration of unsaturated FAs, resulting in a series of reactive species, including $\alpha, \beta$-unsaturated aldehydes such as 4hydroxynonenal (4-HNE) [30]. Hepatocytes are equipped with multiple defense systems that ensure protection against the toxic effects of endogenous and exogenous oxidants and electrophiles to which they are exposed, including (a) phase 1 enzymes that introduce functional groups onto largely hydrophobic organic molecules, such as cytochrome P450 enzymes, whose activity may produce highly reactive products that are toxic to the cell; (b) phase 2 enzymes like glutathione S-transferases and UDP glucuronosyl transferases, which conjugate the products of phase 1 enzymes with hydrophilic groups in order to facilitate their excretion, and antioxidant enzymes like superoxide dismutases, glutathione peroxidase, and catalase, which inactivate ROS; (c) phase 3 efflux transporters that export toxic metabolites acting synergistically with phase 2 enzymes to provide protection against electrophiles and carcinogens; and (d) thiol containing molecules such as glutathione and thioredoxin that function to maintain reducing conditions within the cell and inactivate electrophilic compounds [31]. Importantly, many of these cytoprotective enzymes are encoded by genes containing antioxidant response elements (AREs) in their promoter regions. AREs were initially identified as $5^{\prime}(\mathrm{G} / \mathrm{A}) \mathrm{TGA}(\mathrm{G} / \mathrm{C}) \mathrm{nnnCG}(\mathrm{G} / \mathrm{A}) 3^{\prime}$ [32] and subsequently expanded to $5^{\prime}$ TMAnnRTGAYnnnGCRwwww3', where $\mathrm{M}=$ A or $\mathrm{C}, \mathrm{R}=\mathrm{A}$ or $\mathrm{G}, \mathrm{Y}=\mathrm{C}$ or $\mathrm{T}, \mathrm{W}=\mathrm{A}$ or $\mathrm{T}$, and $\mathrm{S}=\mathrm{G}$ or $\mathrm{C}$ [33].

The ARE is a cis-acting enhancer sequence that mediates transcriptional activation of genes in response to changes in the cellular redox status, such as during increased production of free radical species, or to prooxidant xenobiotics that are thiol reactive and mimic an oxidative insult [34]. Transcription factor nuclear factor-erythroid 2-related factor 2 (Nfe2l2/Nrf2) is a basic leucine zipper transcription factor that regulates transcriptional induction of ARE-containing genes encoding antioxidant enzymes, electrophile-conjugating enzymes, ubiquitin/proteasomes, and chaperone and heat-shock proteins in response to cellular stresses including ROS $[35,36]$. Under normal conditions, 


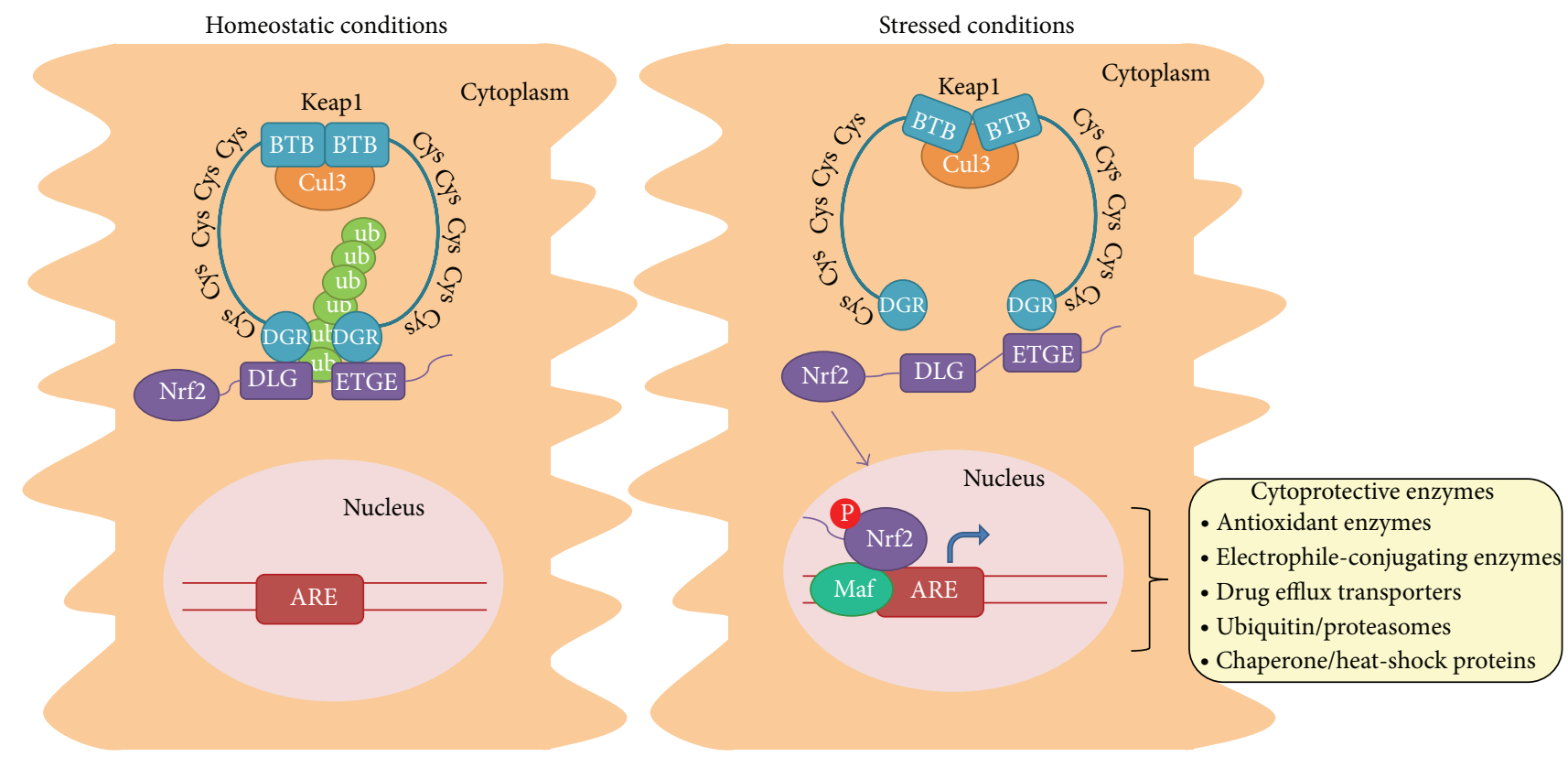

FIGURE 1: Activation of the Keap1-Nrf2-ARE pathway by oxidants/electrophiles. Under homeostatic conditions, Nrf2 is mainly localized in the cytoplasm through an interaction with Keapl and the actin cytoskeleton. Keapl is a five-domain protein consisting of an $\mathrm{N}$-terminal broad complex, Tramtrack and Bric-à-brac (BTB) domain, an intervening region with cysteine (Cys) residues, a C-terminal Kelch domain with double glycine repeats (DGR), and the C-terminal domain. Keapl homodimerizes at the BTB domain, which is also a binding site for Cullin 3 (Cul3). The Keap1 homodimer binds to a single Nrf2 molecule through the ETGE and DLG motifs of Nrf2, each binding to a DGR domain in Keap1. According to the proposed hinge and latch model [13], ETGE is a high-affinity motif ("hinge") whereas DLG is a low-affinity one ("latch"). Keap1 functions as an adaptor protein in the Cul3-based E3 ligase complex, which results in the polyubiquitination (Ub) of the lysine residues situated between the DLG and ETGE motifs, and subsequent proteasomic degradation of Nrf2. Under stressed conditions, the modification of critical cysteine residues of Keap1 destabilizes its binding to the DLG motif of Nrf2, which blocks ubiquitination/proteasomal degradation and allows Nrf2 to escape Keap1 control and translocate into the nucleus. In the nucleus, Nrf2 heterodimerizes with small Maf proteins and promotes the expression of ARE-containing genes involved in cell stress response, drug metabolism, detoxification, and transport. Nrf2 may also be phosphorylated (P) by stress-activated kinases.

Nrf2 is mainly localized in the cytoplasm through an interaction with Kelch ECH associating protein 1 (Keapl) and the actin cytoskeleton. Despite the fact that Nrf2 mRNA is constitutively expressed, Keap1 targets Nrf2 for polyubiquitination and degradation, resulting in a short protein half-life. The binding to and regulation of Nrf2 by Keap1 have been explained by a "hinge and latch model" [13], as described in Figure 1. During exposure to electrophiles or oxidative stress, Keap1 becomes oxidized at critical cysteine residues. As a result, Nrf2 escapes Keapl control and translocates to the nucleus, where it dimerizes with small musculoaponeurotic fibrosarcoma (Maf) proteins and promotes the expression of ARE-containing genes [36-38]. In recent years, it has become apparent that Nrf2 activity is not controlled exclusively through Keapl-mediated proteasomal degradation. In addition to Keap1, Nrf2 protein stability is regulated by another E3 ubiquitin ligase adaptor, $\beta$-transducin repeatcontaining protein $(\beta$-TrCP). Nrf2 degradation independent of Keapl is promoted by glycogen synthase kinase 3 (GSK3), which phosphorylates specific serine residues in the Neh6 domain of Nrf2 corresponding to the $\beta$-TrCP recognition motif [39]. Alternatively, Nrf2 activation may occur as a result of Nrf2 phosphorylation by mitogen activated protein kinase cascade, phosphatidylinositol 3-kinase, protein kinase C, and protein kinase RNA-like endoplasmic reticulum kinase (Perk) $[36,40]$.

In addition to the modulation of protein stability, Nrf2 activity is also regulated at the transcriptional level. Polycyclic aromatic hydrocarbons activate Nrf2 transcription through binding of the heterodimer formed by the aryl hydrocarbon receptor (AhR) and the AhR nuclear translocator to xenobiotic response element-like sequences in the Nrf2 promoter [41]. In certain tumor cells, activation of Nrf2 transcription by Kras oncogene is responsible for increased chemoresistance [42]. Moreover, basal Nrf2 activity seems to be regulated by epigenetic mechanisms and miRNA species, as reviewed elsewhere [43].

\section{Nrf2 Protection against Liver Injury}

As discussed, the Keap1-Nrf2-ARE pathway is activated in response to oxidative/electrophilic stress and regulates the basal and inducible expression levels of a battery of proteins involved in the detoxification of reactive molecules in the cytosol, mitochondria, and ER [30]. Overall, this transcriptional response protects cells against a series of insults and favors cell adaptation/survival $[40,44]$. Cellular survival is also mediated by the UPR, which restores ER homeostasis 
and by the autophagy-lysosomal pathway, which promotes the degradation of proteins and dysfunctional organelles. There is increasing evidence that hepatic steatosis and ER stress are interconnected. This is not surprising since several enzymatic lipogenic pathways are located in the ER, including fatty acid elongation, cholesterol biosynthesis, complex lipid biosynthesis, and assembly of VLDL particles. In fact, abrogation of the UPR results in ER stress-induced development of steatosis [45]. During the UPR, Perk-dependent phosphorylation may also lead to Nrf2 nuclear translocation and increased transcription of Nrf2 target genes [40]. Nrf2 is also activated during autophagy, via interaction of the selective autophagy substrate p62 with the Nrf2 binding site on Keap1 [46]. Accumulation of p62 (as a consequence of a deficiency in autophagy) results in stabilization of Nrf2 and transcriptional activation of Nrf2 target genes [46]. A study by Kwon et al. demonstrated that high steady-state expression of $\mathrm{NAD}(\mathrm{P}) \mathrm{H}$ dehydrogenase, quinone 1, sustained by $\mathrm{p} 62$-induced basal Nrf2 activation, is required to maintain mitochondrial integrity [47]. Other studies have reported that Nrf2 deficiency results in mitochondrial depolarization, reduced ATP production, and decreased rate of oxygen consumption (mitochondrial respiration) [48], as well as less efficient mitochondrial fatty acid oxidation [49].

Several studies have demonstrated that $\mathrm{Nrf} 2^{-/-}$mice are more susceptible to chemical-induced oxidative/electrophilic stress in the liver than wild-type mice [50-52]. Nrf2 protects mice from 2,3,7,8-tetrachlorodibenzo- $p$-dioxin- (TCDD-) induced oxidative damage and steatohepatitis [53] and from hepatic fibrosis caused by chronic treatment with the hepatotoxin carbon tetrachloride [54]. Nrf2-deficient hepatocytes are also more susceptible to the toxicity of excessive iron accumulation [55].

\section{Nrf2 in Liver Regeneration and Aging}

The liver is the only organ in the human body capable of completely regenerating itself after injury. In the regenerating liver, hepatocytes accumulate a significant amount of lipids within lipid droplets, which are mainly composed of triacylglycerol and cholesteryl esters [56-58]. There is growing evidence that $\mathrm{Nrf} 2$ is required for effective tissue repair. Cell regeneration is diminished in hepatectomized Nrf2-null mice, which is associated with increased oxidative stress, reduced insulin/insulin growth factor-1 signaling [59], and reduced expression of the gene encoding a hepatotropic factor, augmenter of liver regeneration [60]. Importantly, the ability of the liver to regenerate after hepatectomy or chemical injury declines with old age [61]. Aging is also associated with increased lipid accumulation in the liver, which may ultimately result in lipotoxicity. Aging has been reported to increase the prevalence of the metabolic syndrome and of NAFLD in the human population and to enhance the progression to NASH and fibrosis [61]. Nrf2 plays an important role in the hepatic aging process. With age, there is a substantial reduction in glutathione (GSH) levels and in the expression and activity of glutamate cysteine ligase, the ratecontrolling enzyme in GSH synthesis. This is accompanied by lower levels of Nrf2 protein and a reduction in Nrf2/ARE binding [62], as well as increased markers of protein and lipid oxidation [63]. Conversely, the liver of aged Nrf2-null mice shows lower free radical reducing activity [64] and GSH synthesis. The reason why aging organisms gradually lose the ability to activate Nrf2 is currently not understood [43], but a decline in Nrf2 signaling is presumed to contribute to the agerelated hepatic oxidative stress. Whether it also contributes to the increased progression from NAFLD to NASH and fibrosis in the elderly is a subject that warrants further investigation.

\section{Nrf2 Regulation of Hepatic Lipid Metabolism}

Besides activating antioxidant and detoxification genes in response to electrophilic or oxidative stress, there is increasing evidence that Nrf2 participates in hepatic fatty acid metabolism (Table 1). A microarray study by Yates et al. [19] showed that the genetic or pharmacological activation of Nrf2 represses the expression of key enzymes involved in fatty acid synthesis, with concomitant reduction in the levels of hepatic lipids. A global analysis of constitutive hepatic protein expression in Nrf2-null and wild-type mice subsequently identified two main groups of Nrf2-regulated proteins. One group comprised proteins involved in phase II drug metabolism and antioxidant defense, for which expression was enhanced in the Nrf2 wild-type animals. The other group corresponded to proteins involved in the synthesis and metabolism of fatty acids and other lipids, and unlike proteins involved in the cellular defense, these proteins were expressed to a higher level in the $\mathrm{Nrf}^{-/-}$animals [20]. It is worth noting that both studies were performed with young adult mice (9-10 weeks of age). Another study performed with mice at 8 weeks of age has reported that, under control diet, mRNAs of sterol regulatory element-binding protein-1c and fatty acid synthase were more expressed in Nrf2-null animals than in the wild-type [18]. However, studies employing older mice (12-25 weeks of age) of the same genetic background (C57BL/6) showed that Nrf2 has little effect in hepatic fatty acid metabolism in animals fed control diets [21-23]. Studies in which mice received HFD have also reported that hepatic lipogenesis is negatively regulated by $\mathrm{Nrf2}[21,24]$. Once again, studies using older mice (at approximately 6 months of age) either failed to detect an effect [23] or identified $\mathrm{Nrf} 2$ as an activator of genes involved in lipid synthesis and uptake (e.g., sterol regulatory element-binding protein, fatty acid synthase, stearoyl-CoA desaturase-1, and peroxisome proliferator-activated receptor- $\gamma$ ) via the induction of nuclear receptor small heterodimer partner (Shp/Nrob2) gene transcription and a downmodulator of genes regulating fatty acid oxidation (e.g., peroxisome proliferator-activated receptor- $\alpha$, acetyl-CoA oxidase, and carnitine palmitoyltransferase $1 \alpha$ ) [25]. It is worth noting that while all studies utilized mice with the C57BL/6 genetic background, the backcross to C57BL/6 was incomplete in the latter study (only 4 backcrosses) [25].

In summary, Nrf2 appears to protect the liver against steatosis by inhibiting lipogenesis and promoting fatty acid oxidation. This may be explained by the activation of 
TABle 1: The effect of Nrf2 activation/deficiency on hepatic lipid accumulation in C57BL/6 mice.

\begin{tabular}{|c|c|c|c|c|}
\hline Gender & Age (weeks) & Hepatic lipid accumulation & Expression of FA synthesis genes & Reference \\
\hline \multicolumn{5}{|c|}{ (a) Standard diet } \\
\hline Male & 8 & Increased steatosis in $\mathrm{Nrf2}^{-/-}$. No difference in TGs & Higher in $\mathrm{Nrf2}^{-/-}$ & [18] \\
\hline Male & 9 & Reduced by Nrf2 activation & Reduced by Nrf 2 activation & [19] \\
\hline Male & 10 & Not reported & Higher in $\mathrm{Nrf}_{2}^{-/-}$ & [20] \\
\hline Male & 12 & No difference in TGs & No difference & [21] \\
\hline Male & 19 & Tendency for increased TGs in Nrf2 $2^{-/-}$ & Tendency for higher levels in $\mathrm{Nrf} 2^{-/-}$ & {$[22]$} \\
\hline Male & 25 & No difference in TGs & No difference & [23] \\
\hline \multicolumn{5}{|c|}{ (b) High-fat diet } \\
\hline Male & 12 & Increased FFAs. No difference in TGs & Higher in $\mathrm{Nrf} 2^{-/-}$ & [21] \\
\hline Male & 19 & Tendency for increased TGs in Nrf2 ${ }^{-/-}$ & No difference & [22] \\
\hline Female & 21 & Reduced lipid content by Nrf2 activation & Reduced by Nrf2 activation & [24] \\
\hline Male & 25 & No difference in TGs & No difference & [23] \\
\hline Male & 26 & Reduced total lipid and TGs in $\mathrm{Nrf}^{-/-}$ & Lower in $\mathrm{Nrf}^{-/-}$ & [25] \\
\hline
\end{tabular}

FFA, free fatty acids; TG, triglyceride.

ARE-containing transcription factors that regulate adipocyte differentiation and adipogenesis (e.g., CCAAT/enhancerbinding protein $\beta$, peroxisome proliferator-activated receptor- $\gamma$, aryl hydrocarbon receptor, and retinoid $\mathrm{X}$ receptor- $\alpha$ ) and by the protection against redox-dependent inactivation of metabolic enzymes (e.g., 3-hydroxy-3methylglutaryl-CoA reductase) [43], as well as by other mechanisms that remain unidentified. Future studies aimed at elucidating the molecular basis of these observations are warranted and authors need to take into account the interference of potential confounding factors. As reviewed herein, the role of Nrf2 on hepatic lipid processing in mice appears to be greatly dependent on the age of the animals, whereas factors such as mouse genetic background or gender do not appear to explain most of the contradictory findings in the literature (Table 1). It is tempting to speculate that the agedependence of the Nrf2 regulation of hepatic lipogenesis may be a consequence of the progressive hepatic accumulation of lipids and/or the attenuation of the antioxidant defenses with age. This is supported by a work of Collins et al., who reported that old $\mathrm{Ldlr}^{-/}$mice (a model that mimics human $\mathrm{NASH}$ and atherosclerosis) suffer increased hepatocyte damage when fed a HFD. When compared with young animals, aged $\mathrm{Ldlr}^{-/-}$mice on HFD showed a decline in the expression of antioxidant genes, which was directly related with a decrease in Nrf2 expression [65]. In addition, these animals displayed more severe hepatic steatosis, along with inflammation and fibrosis (NASH), while their younger counterparts simply developed fatty livers [66]. Studies of Nrf2 activation and/or deficiency in aged mice ( $>50$ weeks of age) would be informative, especially since metabolic disorders are known to have a strong age-dependence.

\section{Nrf2: A Therapeutic Target in NAFLD/NASH?}

Despite its high prevalence in the industrialized world, there is currently no approved pharmacological treatment for
NAFLD. It is crucial to develop and establish new options for the prevention and treatment of NAFLD/NASH. While there is still limited evidence in the literature that Nrf2 is activated in human subjects with NASH [67], Nrf2 deficiency has been repeatedly reported to favor the development of steatohepatitis and fibrosis in rodent models of NASH. In these studies, Nrf2-null animals developed many features of NASH when fed methionine- and choline-deficient (MCD) diet $[18,68,69]$, high-fat diet [70], or high-fat and highcholesterol diet [71]. These studies suggest that Nrf2 plays a key role in limiting the progression of $\mathrm{NASH}$, which could be ascribed to the activation of antioxidative stress response genes but also to the modulation of fatty acid metabolism in hepatocytes. It would be important to assess the efficacy of Nrf2-activating compounds in preventing or treating NAFLD/NASH.

A great variety of thiol-reactive, electrophilic compounds isolated from dietary sources or plants are capable of activating Nrf2/ARE-dependent gene expression through inhibition of Keap1-mediated degradation [72-74]. Nrf2 inducing compounds can be grouped into different categories: (1) phenolic antioxidants (caffeic acid, epigallocatechin-3-gallate, and butylated hydroxyanisol); (2) dithiolethiones (oltipraz, $3 \mathrm{H}$ 1,2-dithiol-3-thione); (3) isothiocyanates (sulforaphane); and (4) triterpenoids (oleanolic acid). The synthetic triterpenoid 2-cyano-3,12-dioxooleana-1,9(11)-dien-28-oic acid (CDDO) and its derivative 1-[2-cyano-3-,12-dioxooleana-1,9(11)-dien28-oyl]imidazole (CDDO-Im) are also potent inducers of Nrf2/ARE signaling $[19,75]$. Studies demonstrating the in vivo chemopreventive and/or neuroprotective properties of oltipraz [74, 76], sulforaphane [77], and CDDO-Im [78] indicate a potential therapeutic usefulness for these Nrf2activating molecules. In addition, new pharmacological Nrf2 activators have been synthesized in recent years, some of which have already entered the clinical trial stage [37, 79], including Protandim, dimethyl fumarate (BG-12), and CDDO-Me (bardoxolone methyl). Protandim (LifeVantage) is a dietary supplement that consists of five low-dose natural Nrf2 activators that activate Nrf2 through multiple 
TABLE 2: The effects of Nrf2 activators in rodent models of NAFLD or NASH.

\begin{tabular}{|c|c|c|c|c|}
\hline Nrf2 activator & Species & Administration route & Reported effect & Reference \\
\hline CDDO-Im & Mouse & Oral & $\begin{array}{l}\text { Prevented HFD-induced increases in body weight, adipose mass, and } \\
\text { hepatic lipid accumulation }\end{array}$ & {$[24]$} \\
\hline $\mathrm{CDDO}-\mathrm{Me}$ & Mouse & Oral & $\begin{array}{l}\text { Reduced hepatic lipid accumulation, proinflammatory cytokine } \\
\text { expression, and lipogenic gene expression in mice fed HFD }\end{array}$ & {$[26]$} \\
\hline Sulforaphane & Mouse & Intraperitoneal & Attenuated hepatic fibrosis induced by bile duct ligation & [27] \\
\hline Sulforaphane & Mouse & Oral & Suppressed oxidative stress and hepatic fibrosis induced by MCD diet & {$[28]$} \\
\hline Oltipraz, NK-252 & Rat & Oral & Attenuated hepatic fibrosis induced by CDAA diet & [29] \\
\hline
\end{tabular}

CDAA, choline-deficient L-amino acid-defined diet; CDDO-Im, 1-[2-cyano-3-,12-dioxooleana-1,9(11)-dien-28-oyl]imidazole; CDDO-Me, 1-[2-cyano-3-,12dioxooleana-1,9(11)-dien-28-oyl]methyl; HFD, high-fat diet; MCD, methionine- and choline-deficient diet.

kinase pathways [80]. BG-12/Tecfidera, an oral therapeutic agent containing dimethyl fumarate (Biogen Idec), has been recently approved for the treatment of multiple sclerosis [81]. Bardoxolone methyl (methyl 2-cyano-3,12-di-oxooleana1,9(11)dien-28-oate) (Reata Pharmaceuticals) was shown to restore kidney function in patients with chronic kidney disease [82], although a recent phase III trial had to be terminated due to adverse effects (http://www.reatapharma.com/).

Whilst there have not been any clinical trials specifically on the effects of Nrf2 activation on liver disease, a number of studies have investigated the effects of Nrf2 activators in rodent models of NAFLD and NASH (Table 2). Oral administration of CDDO-Im, one of the most potent activators of Nrf2 in mouse liver known to date [83], prevented highfat diet induced obesity and hepatic lipid accumulation in wild-type mice but not in Nrf2-null mice [19]. Likewise, oral CDDO-Me administration reduced hepatic lipid accumulation, lipogenic gene expression, and proinflammatory cytokine expression and ameliorated type 2 diabetes in mice fed HFD [26].

Sulforaphane [(-)-1-isothiocyanato-(4R)-methylsulfinylbutane], a natural-occurring isothiocyanate from cruciferous vegetables, strongly induces Nrf2 and ARE-mediated transcription activation through inhibiting Keapl-mediated Nrf2 degradation [84]. Treatment of rats with sulforaphane increases liver mitochondrial antioxidant defenses and protects from prooxidant-induced opening of the mitochondrial permeability transition pore [85]. A study by Oh et al. demonstrated that sulforaphane suppressed transforming growth factor- $\beta$-inducible expression of $\alpha$-smooth muscle actin and profibrogenic genes in an immortalized human hepatic stellate cell line and attenuated bile duct ligation-induced liver fibrosis in mice [27]. In a study by Okada et al., long-term supplementation with sulforaphane suppressed the oxidative stress, inflammation, and hepatic fibrosis induced by $\mathrm{MCD}$ diet in mice [28]. Recently, Shimozono et al. [29] used two chemically distinct types of Nrf2 activator, namely, the dithiolethione oltipraz and a novel biaryl urea compound, termed NK-252 (1-(5-(furan-2-yl)-1,3,4-oxadiazol-2yl)-3-(pyridin-2-ylmethyl)urea). The administration of both agents significantly reduced hepatic fibrosis in rats on a choline-deficient L-amino acid-defined diet. Whilst these studies suggest that Nrf2 activation presents new opportunities for treatment of NASH patients with hepatic fibrosis, it is important to bear in mind that NASH is closely related to overnutrition, insulin resistance, and obesity and not to a deficiency of amino acids such as methionine and choline [86]. Ideally, the use of Nrf2-activating compounds should be tested in animal models of NASH associated with obesity, insulin resistance, or dyslipidemia.

\section{Conclusions}

A shematic overview of the proposed protective roles of Nrf2 in NAFLD is depicted in Figure 2. Rodent studies suggest that Nrf2 has a dual protective role in the progression from hepatic steatosis to steatohepatitis: (i) the negative regulation of genes that promote lipid accumulation in hepatocytes ("first hit"), likely by a combination of mechanisms that remain poorly understood; (ii) the activation of genes that promote the elimination of ROS and electrophiles derived from lipid peroxidation, thus preventing hepatocellular oxidative stress and mitochondrial dysfunction ("second hit"). Apparently, these protective mechanisms become less efficient with aging, which is expected to contribute to disease progression. The nutritional/pharmacological induction of Nrf2 signaling represents as a promising potential new strategy for the prevention and treatment of NAFLD/NASH.

\section{Abbreviations}

ARE: Antioxidant response element

CDDO-Im: 1-[2-Cyano-3-,12-dioxooleana-1,9(11)dien-28-oyl]imidazole

CDDO-Me: 1-[2-Cyano-3-,12-dioxooleana-1,9(11)dien-28-oyl]methyl

ER: $\quad$ Endoplasmatic reticulum

FA: $\quad$ Fatty acid

FFA: $\quad$ Free fatty acid

GSH: Glutathione

HFD: High-fat diet

MCD: $\quad$ Methionine- and choline-deficient

NAFLD: Nonalcoholic fatty liver disease

NASH: $\quad$ Nonalcoholic steatohepatitis

ROS: $\quad$ Reactive oxygen species

TG: $\quad$ Triglyceride

UPR: Unfolded protein response

VLDL: Very low density lipoprotein. 


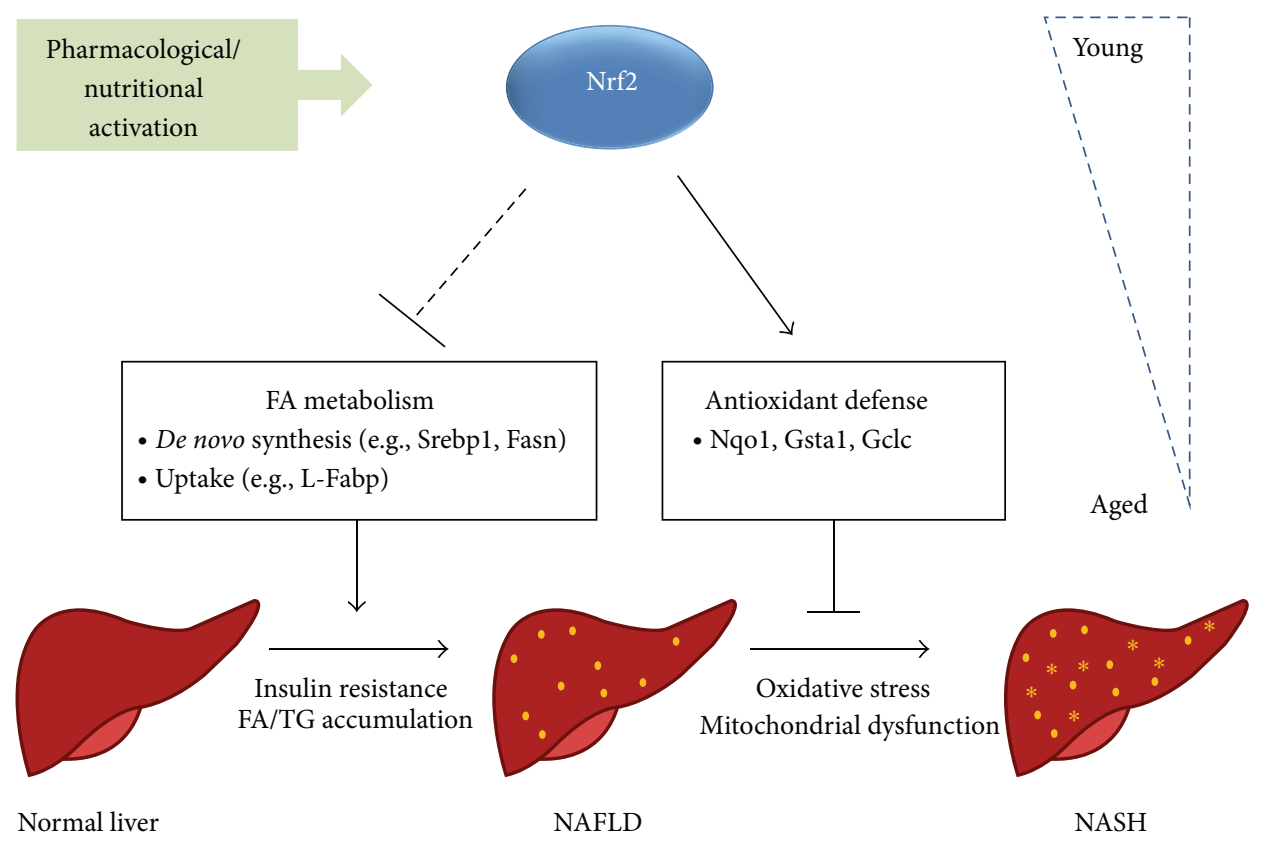

FIGURE 2: Schematic summary of the proposed protective roles of Nrf2 in nonalcoholic fatty liver disease (NAFLD). The progression from the simple accumulation of lipids in the hepatocyte to steatohepatitis (NASH) is depicted. NASH is associated with inflammation, fibrosis, and cirrhosis. The classical understanding is that Nrf2 coordinates the elimination of ROS and electrophiles derived from lipid peroxidation, thus preventing hepatocellular oxidative stress and mitochondrial dysfunction. In addition, there is growing evidence in the literature that Nrf2 regulates fatty acid metabolism by repressing genes that promote lipid accumulation in hepatocytes. In rodents, both mechanisms were shown to inhibit steatohepatitis in an age-dependent manner and can be induced via the pharmacological (e.g., CDDO-Im) or nutritional (e.g., sulforaphane) administration of Nrf2 activators.

\section{Conflict of Interests}

The authors declare no conflict of interests.

\section{Acknowledgments}

This work was supported by FEDER funds through the COMPETE (Operational Competitiveness Programme) and national funds through FCT (Fundação para a Ciência e a Tecnologia) under the project FCOMP-01-0124-FEDER028447 (PTDC/BIM-MET/0739/2012).

\section{References}

[1] J. Huang, J. Borensztajn, and J. K. Reddy, "Hepatic lipid metabolism," in Molecular Pathology of Liver Diseases, S. P. S. Monga, Ed., vol. 5, pp. 133-146, Springer, Berlin, Germany, 2011.

[2] G. Serviddio, F. Bellanti, and G. Vendemiale, "Free radical biology for medicine: learning from nonalcoholic fatty liver disease," Free Radical Biology and Medicine, vol. 65, pp. 952-968, 2013.

[3] Y. Stein and B. Shapiro, "Uptake and metabolism of triglycerides by the rat liver," Journal of Lipid Research, vol. 1, pp. 326-331, 1960.

[4] K. Liu and M. J. Czaja, "Regulation of lipid stores and metabolism by lipophagy," Cell Death and Differentiation, vol. 20, no. 1, pp. 3-11, 2013.

[5] V. Zámbó, L. Simon-Szabó, P. Szelényi, É. Kereszturi, G. Bánhegyi, and M. Csala, "Lipotoxicity in the liver," World Journal of Hepatology, vol. 5, no. 10, pp. 550-557, 2013.
[6] E. L. Seifert, C. Estey, J. Y. Xuan, and M.-E. Harper, "Electron transport chain-dependent and -independent mechanisms of mitochondrial $\mathrm{H} 2 \mathrm{O} 2$ emission during long-chain fatty acid oxidation," The Journal of Biological Chemistry, vol. 285, no. 8, pp. 5748-5758, 2010.

[7] G. Vial, H. Dubouchaud, K. Couturier et al., "Effects of a highfat diet on energy metabolism and ROS production in rat liver," Journal of Hepatology, vol. 54, no. 2, pp. 348-356, 2011.

[8] A. K. Leamy, R. A. Egnatchik, and J. D. Young, "Molecular mechanisms and the role of saturated fatty acids in the progression of non-alcoholic fatty liver disease," Progress in Lipid Research, vol. 52, no. 1, pp. 165-174, 2013.

[9] E. S. Ford, W. H. Giles, and W. H. Dietz, "Prevalence of the metabolic syndrome among US adults: findings from the third National Health and Nutrition Examination Survey," The Journal of the American Medical Association, vol. 287, no. 3, pp. 356-359, 2002.

[10] D. M. Boudreau, D. C. Malone, M. A. Raebel et al., "Health care utilization and costs by metabolic syndrome risk factors," Metabolic Syndrome and Related Disorders, vol. 7, no. 4, pp. 305313, 2009.

[11] K. G. M. M. Alberti, P. Zimmet, and J. Shaw, "Metabolic syndrome-a new world-wide definition. A Consensus Statement from the International Diabetes Federation," Diabetic Medicine, vol. 23, no. 5, pp. 469-480, 2006.

[12] P. Dietrich and C. Hellerbrand, "Non-alcoholic fatty liver disease, obesity and the metabolic syndrome," Best Practice \& Research Clinical Gastroenterology, vol. 28, no. 4, pp. 637-653, 2014. 
[13] K. I. Tong, A. Kobayashi, F. Katsuoka, and M. Yamamoto, “Twosite substrate recognition model for the Keap1-Nrf2 system: a hinge and latch mechanism," The Journal of Biological Chemistry, vol. 387, no. 10-11, pp. 1311-1320, 2006.

[14] C. P. Day and O. F. W. James, "Hepatic steatosis: innocent bystander or guilty party?” Hepatology, vol. 27, no. 6, pp. 1463$1466,1998$.

[15] S.-J. Lee, J. Zhang, A. M. K. Choi, and H. P. Kim, "Mitochondrial dysfunction induces formation of lipid droplets as a generalized response to stress," Oxidative Medicine and Cellular Longevity, vol. 2013, Article ID 327167, 10 pages, 2013.

[16] M. Amir and M. J. Czaja, "Autophagy in nonalcoholic steatohepatitis," Expert Review of Gastroenterology and Hepatology, vol. 5, no. 2, pp. 159-166, 2011.

[17] Z. Tariq, C. J. Green, and L. Hodson, "Are oxidative stress mechanisms the common denominator in the progression from hepatic steatosis towards non-alcoholic steatohepatitis (NASH)?" Liver International, vol. 34, no. 7, pp. e180-e190, 2014.

[18] Y.-K. J. Zhang, R. L. Yeager, Y. Tanaka, and C. D. Klaassen, "Enhanced expression of Nrf2 in mice attenuates the fatty liver produced by a methionine- and choline-deficient diet," Toxicology and Applied Pharmacology, vol. 245, no. 3, pp. 326334, 2010.

[19] M. S. Yates, Q. T. Tran, P. M. Dolan et al., "Genetic versus chemoprotective activation of $\mathrm{Nrf} 2$ signaling: overlapping yet distinct gene expression profiles between Keap1 knockout and triterpenoid-treated mice," Carcinogenesis, vol. 30, no. 6, pp. 1024-1031, 2009.

[20] N. R. Kitteringham, A. Abdullah, J. Walsh et al., "Proteomic analysis of Nrf2 deficient transgenic mice reveals cellular defence and lipid metabolism as primary Nrf2-dependent pathways in the liver," Journal of Proteomics, vol. 73, no. 8, pp. 1612-1631, 2010.

[21] Y. Tanaka, L. M. Aleksunes, R. L. Yeager et al., "NF-E2-related factor 2 inhibits lipid accumulation and oxidative stress in mice fed a high-fat diet," Journal of Pharmacology and Experimental Therapeutics, vol. 325, no. 2, pp. 655-664, 2008.

[22] Y. Tanaka, T. Ikeda, K. Yamamoto, H. Ogawa, and T. Kamisako, "Dysregulated expression of fatty acid oxidation enzymes and iron-regulatory genes in livers of Nrf2-null mice," Journal of Gastroenterology and Hepatology, vol. 27, no. 11, pp. 1711-1717, 2012.

[23] Y.-K. J. Zhang, K. C. Wu, J. Liu, and C. D. Klaassen, "Nrf2 deficiency improves glucose tolerance in mice fed a high-fat diet," Toxicology and Applied Pharmacology, vol. 264, no. 3, pp. 305-314, 2012.

[24] S. Shin, J. Wakabayashi, M. S. Yates et al., "Role of Nrf2 in prevention of high-fat diet-induced obesity by synthetic triterpenoid CDDO-Imidazolide," European Journal of Pharmacology, vol. 620, no. 1-3, pp. 138-144, 2009.

[25] J. Huang, I. Tabbi-Anneni, V. Gunda, and L. Wang, "Transcription factor Nrf2 regulates SHP and lipogenic gene expression in hepatic lipid metabolism," The American Journal of PhysiologyGastrointestinal and Liver Physiology, vol. 299, no. 6, pp. G1211G1221, 2010.

[26] P. K. Saha, V. T. Reddy, M. Konopleva, M. Andreeff, and L. Chan, "The triterpenoid 2-cyano-3,12-dioxooleana-1,9-dien28-oic-acid methyl ester has potent anti-diabetic effects in diet-induced diabetic mice and Lepr ${ }^{d b / d b}$ mice," The Journal of Biological Chemistry, vol. 285, no. 52, pp. 40581-40592, 2010.

[27] C. J. Oh, J.-Y. Kim, A.-K. Min et al., "Sulforaphane attenuates hepatic fibrosis via NF-E2-related factor 2-mediated inhibition of transforming growth factor- $\beta /$ Smad signaling," Free Radical Biology and Medicine, vol. 52, no. 3, pp. 671-682, 2012.

[28] K. Okada, E. Warabi, H. Sugimoto et al., "Nrf2 inhibits hepatic iron accumulation and counteracts oxidative stress-induced liver injury in nutritional steatohepatitis," Journal of Gastroenterology, vol. 47, no. 8, pp. 924-935, 2012.

[29] R. Shimozono, Y. Asaoka, Y. Yoshizawa et al., "Nrf2 activators attenuate the progression of nonalcoholic steatohepatitisrelated fibrosis in a dietary rat models," Molecular Pharmacology, vol. 84, no. 1, pp. 62-70, 2013.

[30] A.-L. Levonen, B. G. Hill, E. Kansanen, J. Zhang, and V. M. Darley-Usmar, "Redox regulation of antioxidants, autophagy, and the response to stress: implications for electrophile therapeutics," Free Radical Biology and Medicine, vol. 71, pp. 196-207, 2014.

[31] A. T. Dinkova-Kostova, W. D. Holtzclaw, and T. W. Kensler, "The role of Keap1 in cellular protective responses," Chemical Research in Toxicology, vol. 18, no. 12, pp. 1779-1791, 2005.

[32] T. H. Rushmore, M. R. Morton, and C. B. Pickett, "The antioxidant responsive element: activation by oxidative stress and identification of the DNA consensus sequence required for functional activity," Journal of Biological Chemistry, vol. 266, no. 18, pp. 11632-11639, 1991.

[33] W. W. Wasserman and W. E. Fahl, "Functional antioxidant responsive elements," Proceedings of the National Academy of Sciences of the United States of America, vol. 94, no. 10, pp. 53615366, 1997.

[34] T. Nguyen, P. J. Sherratt, and C. B. Pickett, "Regulatory mechanisms controlling gene expression mediated by the antioxidant response element," Annual Review of Pharmacology and Toxicology, vol. 43, pp. 233-260, 2003.

[35] J.-M. Lee, M. J. Calkins, K. Chan, Y. W. Kan, and J. A. Johnson, "Identification of the NF-E2-related factor-2-dependent genes conferring protection against oxidative stress in primary cortical astrocytes using oligonucleotide microarray analysis," The Journal of Biological Chemistry, vol. 278, no. 14, pp. 12029-12038, 2003.

[36] T. W. Kensler, N. Wakabayashi, and S. Biswal, "Cell survival responses to environmental stresses via the Keap1-Nrf2-ARE pathway," Annual Review of Pharmacology and Toxicology, vol. 47, pp. 89-116, 2007.

[37] T. Suzuki, H. Motohashi, and M. Yamamoto, "Toward clinical application of the Keap1-Nrf2 pathway," Trends in Pharmacological Sciences, vol. 34, no. 6, pp. 340-346, 2013.

[38] E. Kansanen, H.-K. Jyrkkänen, and A.-L. Levonen, "Activation of stress signaling pathways by electrophilic oxidized and nitrated lipids," Free Radical Biology and Medicine, vol. 52, no. 6, pp. 973-982, 2012.

[39] P. Rada, A. I. Rojo, S. Chowdhry, M. McMahon, J. D. Hayes, and A. Cuadrado, "SCF/ $\beta$-TrCP promotes glycogen synthase kinase 3-dependent degradation of the Nrf2 transcription factor in a Keapl-independent manner," Molecular and Cellular Biology, vol. 31, no. 6, pp. 1121-1133, 2011.

[40] S. B. Cullinan and J. A. Diehl, "Coordination of ER and oxidative stress signaling: the PERK/Nrf2 signaling pathway," The International Journal of Biochemistry \& Cell Biology, vol. 38, no. 3, pp. 317-332, 2006.

[41] W. Miao, L. Hu, P. J. Scrivens, and G. Batist, “Transcriptional regulation of NF-E2 p45-related factor (NRF2) expression by 
the aryl hydrocarbon receptor-xenobiotic response element signaling pathway: direct cross-talk between phase I and II drugmetabolizing enzymes," The Journal of Biological Chemistry, vol. 280, no. 21, pp. 20340-20348, 2005.

[42] S. Tao, S. Wang, S. J. Moghaddam et al., "Oncogenic KRAS confers chemoresistance by upregulating NRF2," Cancer Research, vol. 74, no. 24, pp. 7430-7441, 2014.

[43] J. D. Hayes and A. T. Dinkova-Kostova, "The Nrf2 regulatory network provides an interface between redox and intermediary metabolism," Trends in Biochemical Sciences, vol. 39, no. 4, pp. 199-218, 2014.

[44] W. O. Osburn and T. W. Kensler, "Nrf2 signaling: an adaptive response pathway for protection against environmental toxic insults," Mutation Research-Reviews in Mutation Research, vol. 659, no. 1-2, pp. 31-39, 2008.

[45] H. Malhi and R. J. Kaufman, "Endoplasmic reticulum stress in liver disease," Journal of Hepatology, vol. 54, no. 4, pp. 795-809, 2011.

[46] M. Komatsu, H. Kurokawa, S. Waguri et al., "The selective autophagy substrate p62 activates the stress responsive transcription factor Nrf2 through inactivation of Keapl," Nature Cell Biology, vol. 12, no. 3, pp. 213-223, 2010.

[47] J. Kwon, E. Han, C.-B. Bui et al., "Assurance of mitochondrial integrity and mammalian longevity by the p62-Keap1-Nrf2Nqol cascade," EMBO Reports, vol. 13, no. 2, pp. 150-156, 2012.

[48] K. M. Holmstrom, L. Baird, Y. Zhang et al., "Nrf2 impacts cellular bioenergetics by controlling substrate availability for mitochondrial respiration," Biology Open, vol. 2, no. 8, pp. 761770, 2013

[49] M. H. R. Ludtmann, P. R. Angelova, Y. Zhang, A. Y. Abramov, and A. T. Dinkova-Kostova, "Nrf2 affects the efficiency of mitochondrial fatty acid oxidation," Biochemical Journal, vol. 457, no. 3, pp. 415-424, 2014.

[50] L. M. Aleksunes and J. E. Manautou, "Emerging role of Nrf2 in protecting against hepatic and gastrointestinal disease," Toxicologic Pathology, vol. 35, no. 4, pp. 459-473, 2007.

[51] C. D. Klaassen and S. A. Reisman, "Nrf2 the rescue: effects of the antioxidative/electrophilic response on the liver," Toxicology and Applied Pharmacology, vol. 244, no. 1, pp. 57-65, 2010.

[52] J. Liu, K. C. Wu, Y.-F. Lu, E. Ekuase, and C. D. Klaassen, "NRF2 protection against liver injury produced by various hepatotoxicants," Oxidative Medicine and Cellular Longevity, vol. 2013, Article ID 305861, 8 pages, 2013.

[53] H. Lu, W. Cui, and C. D. Klaassen, "Nrf2 protects against 2,3,7,8tetrachlorodibenzo-p-dioxin (TCDD)-induced oxidative injury and steatohepatitis," Toxicology and Applied Pharmacology, vol. 256, no. 2, pp. 122-135, 2011.

[54] W. Xu, C. Hellerbrand, U. A. Köhler et al., "The Nrf2 transcription factor protects from toxin-induced liver injury and fibrosis," Laboratory Investigation, vol. 88, no. 10, pp. 1068-1078, 2008.

[55] S. Silva-Gomes, A. G. Santos, C. Caldas et al., "Transcription factor NRF2 protects mice against dietary iron-induced liver injury by preventing hepatocytic cell death," Journal of Hepatology, vol. 60, no. 2, pp. 354-361, 2014.

[56] L. B. M. Tijburg, C. B. Nyathi, G. W. Meijer, and M. J. H. Geelen, "Biosynthesis and secretion of triacylglycerol in rat liver after partial hepatectomy," The Biochemical Journal, vol. 277, no. 3, pp. 723-728, 1991.

[57] E. P. Newberry, S. M. Kennedy, Y. Xie et al., "Altered hepatic triglyceride content after partial hepatectomy without impaired liver regeneration in multiple murine genetic models," Hepatology, vol. 48, no. 4, pp. 1097-1105, 2008.

[58] I. García-Arcos, P. González-Kother, P. Aspichueta, Y. Rueda, B. Ochoa, and O. Fresnedo, "Lipid analysis reveals quiescent and regenerating liver-specific populations of lipid droplets," Lipids, vol. 45, no. 12, pp. 1101-1108, 2010.

[59] T. A. Beyer, W. Xu, D. Teupser et al., "Impaired liver regeneration in Nrf2 knockout mice: Role of ROS-mediated insulin/IGF1 resistance," EMBO Journal, vol. 27, no. 1, pp. 212-223, 2008.

[60] R. Dayoub, A. Vogel, J. Schuett et al., "Nrf2 activates augmenter of liver regeneration (ALR) via antioxidant response element and links oxidative stress to liver regeneration," Molecular Medicine, vol. 19, no. 1, pp. 237-244, 2013.

[61] F. Sheedfar, S. D. Biase, D. Koonen, and M. Vinciguerra, "Liver diseases and aging: friends or foes?” Aging Cell, vol. 12, no. 6, pp. 950-954, 2013.

[62] J. H. Suh, S. V. Shenvi, B. M. Dixon et al., "Decline in transcriptional activity of Nrf2 causes age-related loss of glutathione synthesis, which is reversible with lipoic acid," Proceedings of the National Academy of Sciences of the United States of America, vol. 101, no. 10, pp. 3381-3386, 2004.

[63] P.-H. Shih and G.-C. Yen, "Differential expressions of antioxidant status in aging rats: the role of transcriptional factor Nrf2 and MAPK signaling pathway," Biogerontology, vol. 8, no. 2, pp. 71-80, 2007.

[64] A. Hirayama, K. Yoh, S. Nagase et al., "EPR imaging of reducing activity in Nrf2 transcriptional factor-deficient mice," Free Radical Biology and Medicine, vol. 34, no. 10, pp. 1236-1242, 2003.

[65] A. R. Collins, C. J. Lyon, X. Xia et al., "Age-accelerated atherosclerosis correlates with failure to upregulate antioxidant genes," Circulation Research, vol. 104, no. 6, pp. e42-e54, 2009.

[66] A. A. Gupte, C. J. Lyon, and W. A. Hsueh, "Nuclear factor (erythroid-derived 2)-like-2 factor (Nrf2), a key regulator of the antioxidant response to protect against atherosclerosis and nonalcoholic steatohepatitis," Current Diabetes Reports, vol. 13, no. 3, pp. 362-371, 2013.

[67] R. N. Hardwick, C. D. Fisher, M. J. Canet, A. D. Lake, and N. J. Cherrington, "Diversity in antioxidant response enzymes in progressive stages of human nonalcoholic fatty liver disease," Drug Metabolism and Disposition, vol. 38, no. 12, pp. 2293-2301, 2010.

[68] H. Sugimoto, K. Okada, J. Shoda et al., "Deletion of nuclear factor-E2-related factor-2 leads to rapid onset and progression of nutritional steatohepatitis in mice," American Journal of Physiology: Gastrointestinal and Liver Physiology, vol. 298, no. 2, pp. G283-G294, 2010.

[69] S. Chowdhry, M. H. Nazmy, P. J. Meakin et al., "Loss of Nrf2 markedly exacerbates nonalcoholic steatohepatitis," Free Radical Biology \& Medicine, vol. 48, no. 2, pp. 357-371, 2010.

[70] C. Wang, Y. Cui, C. Li et al., "Nrf2 deletion causes "benign" simple steatosis to develop into nonalcoholic steatohepatitis in mice fed a high-fat diet," Lipids in Health and Disease, vol. 12, no. 1, article 165, 2013.

[71] K. Okada, E. Warabi, H. Sugimoto et al., "Deletion of Nrf2 leads to rapid progression of steatohepatitis in mice fed atherogenic plus high-fat diet," Journal of Gastroenterology, vol. 48, no. 5, pp. 620-632, 2013.

[72] Y. Zhang and G. B. Gordon, "A strategy for cancer prevention: stimulation of the Nrf2-ARE signaling pathway," Molecular Cancer Therapeutics, vol. 3, no. 7, pp. 885-893, 2004. 
[73] A. T. Dinkova-Kostova, C. Abeygunawardana, and P. Talalay, "Chemoprotective properties of phenylpropenoids, bis(benzylidene)cycloalkanones, and related Michael reaction acceptors: correlation of potencies as phase 2 enzyme inducers and radical scavengers," Journal of Medicinal Chemistry, vol. 41, no. 26, pp. 5287-5296, 1998.

[74] M. Ramos-Gomez, M.-K. Kwak, P. M. Dolan et al., "Sensitivity to carcinogenesis is increased and chemoprotective efficacy of enzyme inducers is lost in Nrf2 transcription factor-deficient mice," Proceedings of the National Academy of Sciences of the United States of America, vol. 98, no. 6, pp. 3410-3415, 2001.

[75] K. Liby, T. Hock, M. M. Yore et al., "The synthetic triterpenoids, CDDO and CDDO-imidazolide, are potent inducers of heme oxygenase-1 and Nrf2/ARE signaling," Cancer Research, vol. 65, no. 11, pp. 4789-4798, 2005.

[76] K. Iida, K. Itoh, Y. Kumagai et al., "Nrf2 is essential for the chemopreventive efficacy of oltipraz against urinary bladder carcinogenesis," Cancer Research, vol. 64, no. 18, pp. 6424-6431, 2004.

[77] J. Zhao, A. N. Moore, J. B. Redell, and P. K. Dash, "Enhancing expression of Nrf2-driven genes protects the blood-brain barrier after brain injury," The Journal of Neuroscience, vol. 27, no. 38, pp. 10240-10248, 2007.

[78] M. S. Yates, M.-K. Kwak, P. A. Egner et al., "Potent protection against aflatoxin-induced tumorigenesis through induction of Nrf2-regulated pathways by the triterpenoid 1-[2-cyano-3-,12dioxooleana-1, 9(11)-dien-28-oyl]imidazole," Cancer Research, vol. 66, no. 4, pp. 2488-2494, 2006.

[79] A. M. Bataille and J. E. Manautou, "Nrf2: a potential target for new therapeutics in liver disease," Clinical Pharmacology \& Therapeutics, vol. 92, no. 3, pp. 340-348, 2012.

[80] S. K. Nelson, S. K. Bose, G. K. Grunwald, P. Myhill, and J. M. McCord, "The induction of human superoxide dismutase and catalase in vivo: a fundamentally new approach to antioxidant therapy," Free Radical Biology and Medicine, vol. 40, no. 2, pp. 341-347, 2006.

[81] L. Kappos, R. Gold, D. H. Miller et al., "Efficacy and safety of oral fumarate in patients with relapsing-remitting multiple sclerosis: a multicentre, randomised, double-blind, placebo-controlled phase IIb study," The Lancet, vol. 372, no. 9648, pp. 1463-1472, 2008.

[82] P. E. Pergola, P. Raskin, R. D. Toto et al., "Bardoxolone methyl and kidney function in CKD with type 2 diabetes," The New England Journal of Medicine, vol. 365, no. 4, pp. 327-336, 2011.

[83] M. S. Yates, M. Tauchi, F. Katsuoka et al., "Pharmacodynamic characterization of chemopreventive triterpenoids as exceptionally potent inducers of Nrf2-regulated genes," Molecular Cancer Therapeutics, vol. 6, no. 1, pp. 154-162, 2007.

[84] W.-S. Jeong, Y.-S. Keum, C. Chen et al., "Differential expression and stability of endogenous nuclear factor E2-related factor 2 (Nrf2) by natural chemopreventive compounds in HepG2 human hepatoma cells," Journal of Biochemistry and Molecular Biology, vol. 38, no. 2, pp. 167-176, 2005.

[85] T. Greco, J. Shafer, and G. Fiskum, "Sulforaphane inhibits mitochondrial permeability transition and oxidative stress," Free Radical Biology and Medicine, vol. 51, no. 12, pp. 2164-2171, 2011.

[86] A. Nakamura and Y. Terauchi, "Lessons from mouse models of high-fat diet-induced NAFLD," International Journal of Molecular Sciences, vol. 14, no. 11, pp. 21240-21257, 2013. 

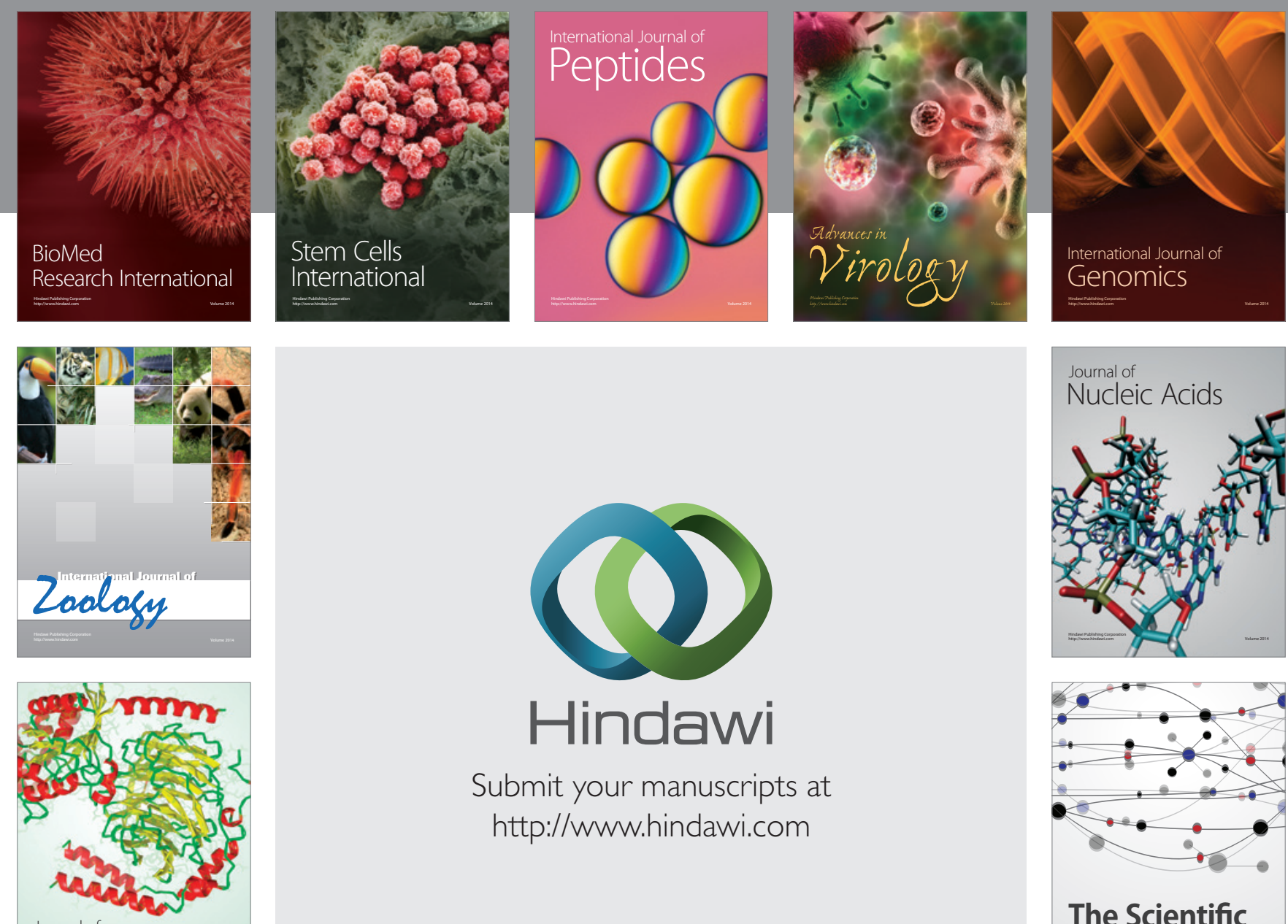

Submit your manuscripts at

http://www.hindawi.com

Journal of
Signal Transduction
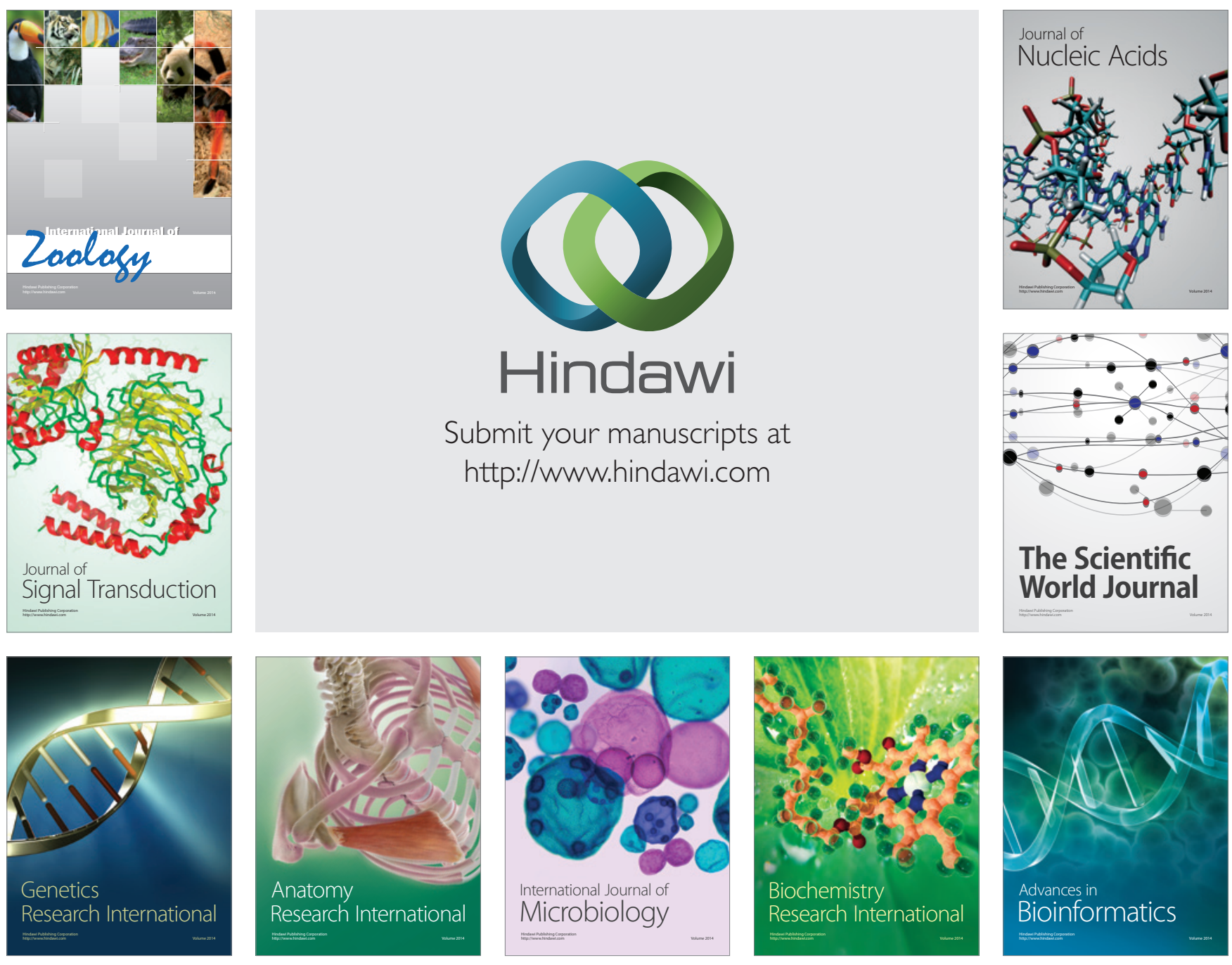

The Scientific World Journal
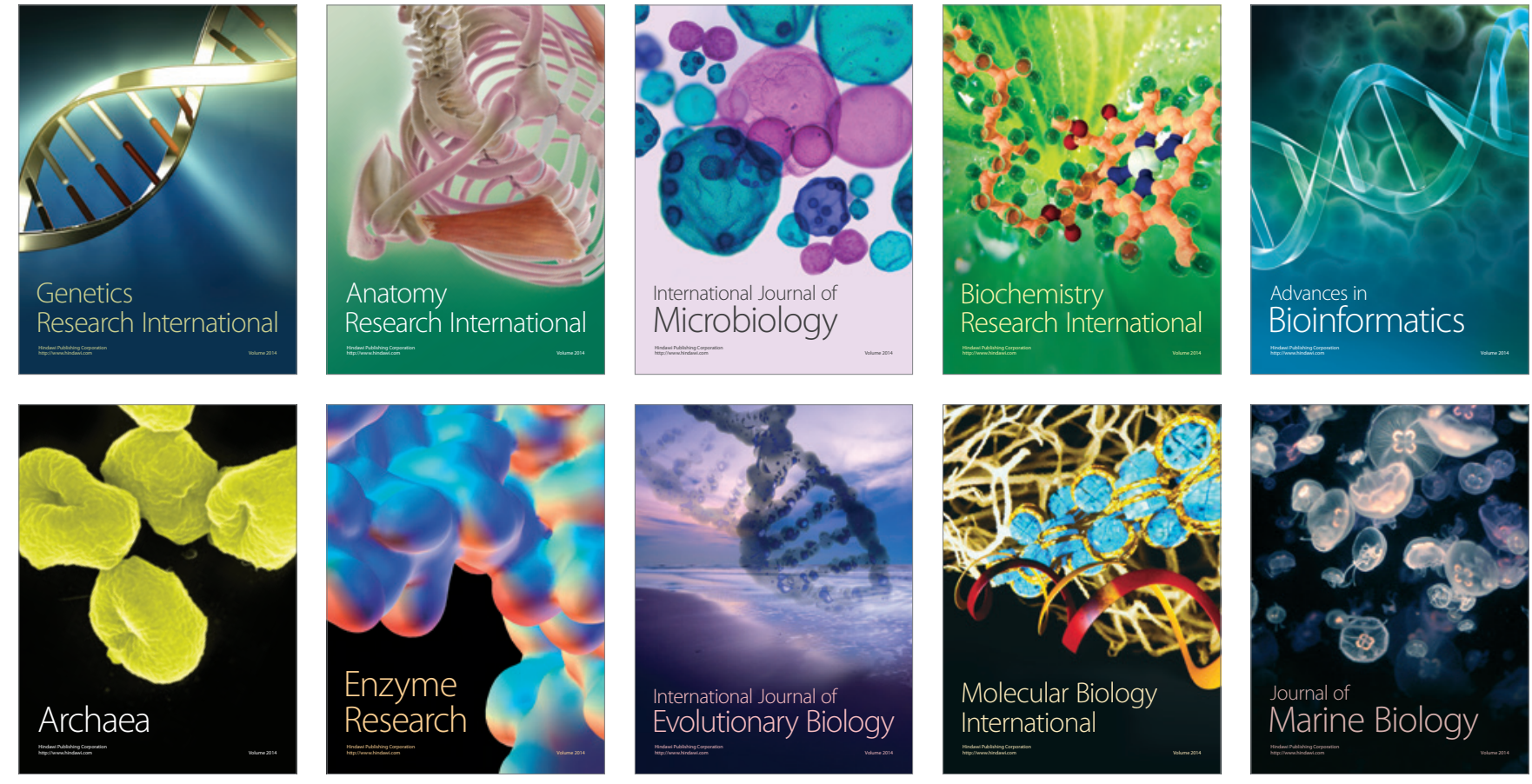